\title{
An IMS-Learning Design Editor for a Higher Education Blended Learning Scenario
}

\author{
García-Robles, Rocío $\quad$ Ferrer, Laura Cagigas, Daniel \\ Computer Architecture and Technology Department, University of Seville \\ rocio@atc.us.es laura@atc.us.es dcagigas@atc.us.es
}

\begin{abstract}
The IMS-Learning Design has been developed to support the creation of reusable and pedagogically neutral learning scenarios and content. Although it is especially suitable for eLearning, there is a lot of interest on using it in higher education blended learning scenarios. However there are some related key issues which must be managed such as cultural bias and the need for expensive human resources to design and develop specification compliant units of learning. They can be addressed by the design of ad-hoc editors supporting concrete learning design units of learning. We suggest some solutions to overcome these limitations, based on our experience designing the user interface of an IMS-LD compliant editor, GDUS+. We also explain our user centering approach, and give some conclusions about the benefits of using IMS-LD.
\end{abstract}

\section{Introduction}

The Learning Design (LD) specification aims to represent the 'learning design' of 'units of learning' (UoL), in a semantic, formal and machine interpretable way [1].

A UoL can be any instructional or learning event of any granularity, e.g. a course, a workshop, a lesson or an informal learning event. A 'learning design' is defined as the description of the teaching-learning process that takes place in the UoL [2]. The key principle in $\mathrm{LD}$ is that it represents the learning activities and the support activities that are performed by different persons (learners, teachers) in the context of a UoL. These activities can refer to different learning objects that are used during the performance of the activities (e.g. books, articles, software applications, pictures), and it can refer to services (e.g. forums, chats, wiki's) that are used to collaborate and to communicate in the teaching-learning process [2].
The Learning Design specification can be seen from (at least) four different perspectives: (1) An educational modeling language; (2) an eLearning methodology; (3) a set of applications; (4) and an interoperability specification [3].

The afore-mentioned specification has been developed to meet some specific requirements: Completeness, Pedagogical expressiveness, Personalization and Compatibility [4].

In the framework of the department of the University of Seville (US) in which the GDUS+ has been designed, completeness and pedagogical expressiveness are considered key issues for several reasons. Firstly, because blended learning solutions demand innovative pedagogic approaches, and IMSLD can support them. Secondly, because the use of GDUS+ tool could be extended in the future to other subjects taught by other departments whose educational approach may differ, so pedagogical flexibility and neutrality are required.

GDUS+ stands for "Guías Docentes de la Universidad de Sevilla". It is an extension of a tool of mandatory use at University of Seville (GDUS), which supports the teachers' community to describe teaching planning. GDUS+ can be considered as a specific purpose tool, distant from specification according to the LD tool design graphic [2].

\section{Special requirements for designing an IMS-LD editor for a higher education blended learning scenario}

Before taking the decision of designing an ad-hoc editor, we were thinking about using other already available tools, such as: RELOAD [5], MOTPlus [6], etc. We were also looking for innovative approaches such the ones proposed by the following tools: DBATLD [7], ModX [8] and WebLD [9] .

The most innovative ones are still in an experimental stage. The other analyzed editing tools are very powerful because they support largely several 
levels of the IMS-LD specification, and most of them are general purpose authoring tools. But, on the other hand, they are still very demanding for most of those teachers, especially in terms of usability. Additionally, we found that there is a cultural bias towards using these types of advanced tools in the department. Moreover we also identified the following drawbacks related with our goal of using IMS-LD in a blended learning scenario. We took the corresponding decisions, as presented bellow:

1) The teachers working in the department are willing to innovate their teaching practices, as long as the increase in their workload is kept to a minimum. As they have to combine teaching and research activities, so the additional workload of implementing blended learning must be minimized. Bearing in mind these issues, one best solution may be to use IMS-LD just as an e-learning methodology In fact, the department used that approach in a pilot experience for a Problem Based Learning scenario, but the conclusions are out of scope of the current paper. Another best solution is to design a user-friendly editor such as GDUS+ which supports just a specific pedagogic model.

2) The initial effort needed to create a pedagogical scenario using that specification is likely to be greater than when teachers think in terms of content and split responsibilities up among the teachers involved. Therefore we decided to create an ad-hoc User Interface (UI) for the most demanded pedagogical model consisting of a single-learner, instructional approach. We also took the decision of keeping the assessment facility as simple as possible, making correction and feedback not automated, but just guided. So the student should make the assessment trial, then compare it to the possible solution(s), and finally to read a table of results and the corresponding recommendations.

3) On one hand, a very high level of detail (related to the granularity of the specification) is required to make a machine-readable version of the UoL. On the other hand, according to experts in this field, editing tools should be distant from the specification and specific purpose for teachers [2]. This is especially true in the case of a blended learning environment, as a consequence of the facts explained previously in bullets one and two. The specification is divided into three parts (levels A, B and C), to make the task of implementation simpler [3]. Tackling the three levels of the specification in a blended learning environment (with limited human resources) still poses a challenge to teachers. Therefore, we decided GDUS+ would only support level A, at least initially.

In summary: (a) an ad-hoc tool has been designed to be adapted to the usual users' cultural environment; (b) for just a single-learner instructional pedagogic model; (c) supporting only the level A of the IMS-LD specification; (d) keeping the assessment facilities to the simplest canonical version; and (e) doing the specification details as invisible as possible.

\section{The UI design process}

Firstly, the users' usual workflow was analyzed, mapping the teaching dynamics to the IMS-LD level A specification. As a consequence, several design decisions were taken. For example, one requirement was to create every activity-structure reference using the name of the corresponding lesson specified by the teacher in the GDUS general tool. GDUS+ supports the authoring of one or more courses for the same matter. But, although the standard supports several LD methods, only one was decided to be supported for each course. There is an act (role-parts) for each lesson. Resources are managed as feedback of an act or as a learning object belonging to an environment (resource type and the path or URL are mandatory). In every course, one or more teachers are able to be involved. The description of the use of services in the IMS-LD compliant version is not supported in GDUS+.

Secondly, main usability criteria were established. According to Nielsen [14], usability has many aspects, and is often associated with the following five attributes: (a) Easy to learn; (b) Efficient to use; (c) Easy to remember; (d) Few errors; and (e) Pleasant to use.

From a usability engineering perspective, one should not necessarily pay equal attention to all usability attributes. Some are more important than others and should be the focus of the development efforts throughout the iterative design. In some cases one might even accept lower scores on certain usability metrics as long as their values remain above some minimally acceptable value [14].

Therefore, in the design of GDUS+ the usability attributes mainly promoted were (a), (b) and (e).

Thirdly, according to a usability engineering approach, models and methods of Usage-Centered Design (UCD) [12] were adopted for the design of the UI of GDUS+. Besides, as it is explained in next section, some metrics for the validation of the different prototypes and user evaluation were adopted. So, for validation of static UI prototypes expert evaluation and users reviews were applied. For the interactive UI prototype, thinking aloud and video recorded were implemented. For users evaluation a heuristic procedure as well as structural and semantic metrics were used. 
The UCD method corresponds to a part of an evolutionary iterative software engineering life-cycle. Its logic process is compound of four models: (1)User Roles (not necessarily matching the actual actors); (2) Task Cases; (3)Abstract Prototype; and (4)Visual and Interaction Design. All those models are developed in an iterative Design/ Prototyping/ ValidationEvaluation cycle.

In GDUS+ case, the User Roles model distinguishes four main roles (Student, Coordinator, Teacher, Tutor). The creation of sub-roles is also supported.

The Task Cases model includes several requirements related to: (A) the context of the GDUS general tool; (B) and the use of the IMS-LD specification. For example, title, objectives, prerequisites of a course, and lessons schedule can be imported from the original GDUS forms. They are filled in by the teacher, avoiding workload increase by duplication of tasks.

Concerning the Content models, firstly different static Abstract Prototypes were designed using post-its. They were used to describe the different interaction contexts for every identified task case (according to the UI Visibility principle [12]). Secondly, the Visual and Interaction Prototypes were developed using Microsoft Visio for the design and Microsoft PowerPoint to simulate the UI dynamics. A navigation map was also implemented as a part of the prototype.

As a result of all that design process, the final visual prototype has the following features: (1) there are two operational modes: advanced and guided (wizard); (2) it is supported to filled up some values by default, saving a lot of time to teachers; (3) there are two types of help strategies. The implicit help is supported by the contextual description of every active UI element, as well as visual guiding of the different steps for authoring a course. The explicit help is implemented by an online table of contents.

\section{Validation of the prototype and user evaluation}

As it was previously commented, GDUS + is performed through a UCD. This means to take into account two important issues: (A) the necessity to iterate some steps in order to improve the application and its usability; (B) and the user relevance during the overall design process.

Thus, the initial validation involved a group of users and was mainly centered on contents. At first, the project in its current phase was introduced to users. Then, Abstract Prototypes were described and explained. Finally, their opinions and comments were collected. Three different Abstract Prototypes were proposed to users. The first one was a stand alone application. The other two were integrated prototypes (partial and fully integrated) into the GDUS tool.

The first prototype was rapidly discarded by users because they considered that was not useful to establish another different way to input data. They decided that the most suitable was the partially integrated one. In this prototype course creation appears as an option in the GDUS general tool. This decision was justified, on one hand, because the creation of virtual courses was considered as a new functionality. On the other hand, the total integrated prototype was considered confusing and less usable because it integrated the course creation through the overall GDUS tool.

Some other conclusions are listed below:

- A distinction between course activities and extra activities must be made (e.g. test of previous knowledge may be available at the beginning of the course).

- An 'advance user profile' (or wizards) should be included.

- Establishment of some kind of 'options by default' facilities before course creation.

The second validation was focused on the structure and the visual organization of GDUS+. The prototype under evaluation offered a passive UI (without real functionality) and high fidelity (close to the final fully implemented UI). Users were asked again to fulfill some tasks in the same manner as they would do with the operational application. Comments and acts were recorded in order to identify possible problems. Some of the corrections that were needed are the following ones:

- Help access had to be moved.

- Tasks order changed their position and/or the way they were selected.

- Some terms names had to be changed.

- Informative tags and texts were added at the beginning of each section.

These results were also obtained from two more complementary evaluations:

The first one consisted in a usability heuristic evaluation. Usability heuristic evaluations are based on ten points proposed by Nielsen in [14]. Heuristic evaluation is usually performed by experts. However, in this case, that evaluation was undertaken also by users.

The second extra evaluation was based on usability metrics. They can be defined as software quality metrics in order to achieve better usability solutions, and they are divided into three groups: 'preference metrics', 'performance metrics' and 'predictive metrics' [12]. For the preference metrics a 'System 
Usability Scale' (SUS) questionnaire was given to users.

Moreover, specific user interface design metrics were also used. They are divided in three groups: structural, semantic and procedural metrics. Structural and semantic metrics are centered in interface contents, while procedural metrics deal with interface tasks. For structural metrics 'Visual Components Alignment', 'Number of Adjacent Screens/Dialogs', and 'Layout Uniformity' were implemented. For semantic metrics 'Visual Coherence' was checked. Those metrics were applied in order to compare the prototype before the second validation and the final prototype. Both studies confirmed that final prototype had improvements when comparing with the initial prototype.

\section{The support of GDUS+ for teachers' workflow from the User Interface perspective}

The IMS-Learning Design (IMS-LD) [10] implies a strong orientation towards reusability and interoperability. But another feature is strong teacher support: we think tools should support the usual workflow of UoL preparation, and the user should not need to know anything about the standard for working.

Supporting the principles of conceptual design as defined by [11], the General User Interface (GUI) features a multiple-window paradigm in such a way that each window encapsulates information related to only one part of the standard. It also allows users to decide when, and how interact with what information. It supports varying user roles, and the standard specification structure.

GDUS+ has been designed according to an authoring oriented approach, trying to keep the specification complexity invisible to the user. By contrast, many already created learning authoring tools complying with IMS LD specification have GUIs which resemble very closely specification related concepts such as content packaging process and metatagging. This approach may be closer to the educational publishing industry way of doing, but it is far away from normal teaching practices, especially in blended learning scenarios.

For that purpose, the application was designed taking usage-centered and usability approaches. A few factors were identified in this success, and are suggested as UI recommendations.

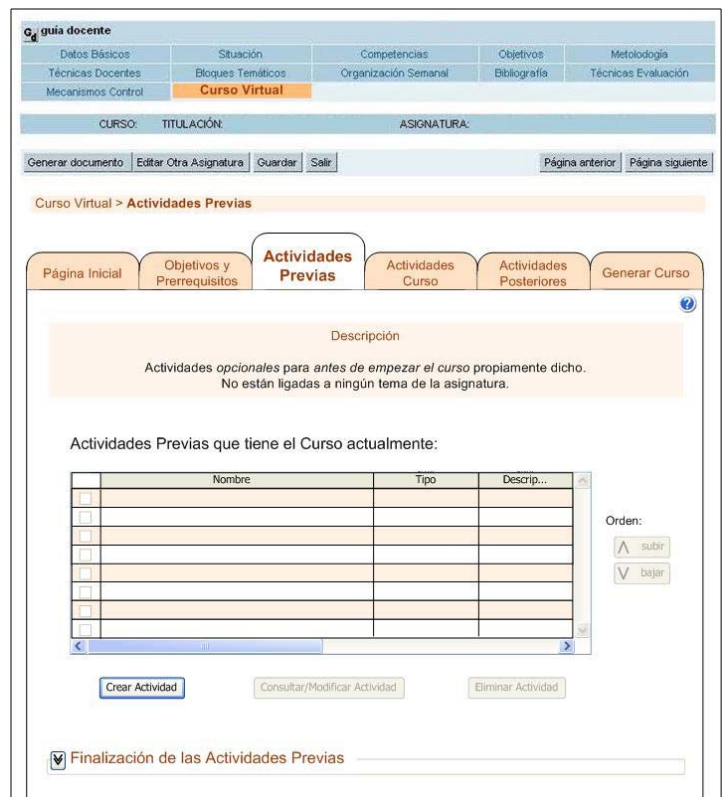

Figure 1: GDUS+

Firstly, the interface reflects the essential structure of the standard grouping the elements according to their functionality; standardization requires that the specification elements and their corresponding relationships must be reflected in the GUI design. Secondly, the terminology used is not specialized; usage requires to translate the terminology and to enlarge the information available in the specification data model. Thirdly, the GUI reflects information supporting teachers' usual workflow of units of learning preparation, supporting and promoting to reuse, recombine, share and visualize content. Fourthly, in order to break the cultural bias, our tool is integrated into the corporative tool (GDUS). The use of that corporative tool is compulsory for all teachers, while GDUS+ use is not. But the GUI design of our tool is homogeneous to the compulsory tool in order to facilitate teachers to easily learn how to use the tool.

\section{Benefits of using IMS-LD}

Some of the benefits of adopting an IMS-LD approach by using an editor such as GDUS+ are:

1) It promotes the use of a roles model for supporting the distinction between possible roles and real actors. The explicit distinction between roles addresses the division of responsibilities improving the coordination and supporting tasks, and making the solution easily scalable regardless of the number of teachers.

2) It promotes the structuring of activities such as preparatory work, logistical tasks, sequential activities, etc. Therefore the process dynamics -which 
is a key issue in the teaching/learning experience--, is made explicit. Moreover it helps to distinguish between learning and support activities. Although it is usual to take into account such a distinction, it should be made explicit in the pedagogical design from the very outset to prevent the coordinator from becoming overloaded.

3) The allocation of resources and environments is also made explicit when designing a pedagogical scenario using LD. In this way, it is easier to think in terms of scalability and feasibility when the allocation is clearly defined from the outset.

4) Reusability of the learning design is promoted. Of course, teachers must review the design and implementation of the scenario and adapt it to the new running or subject, but the design and adaptation costs are estimated to be very low once the course or UoL has been designed and implemented.

5) There are a few main benefits related to the use of tools like GDUS+ in blended learning. Firstly, they support teachers in making explicit description of the pedagogic model (in this case, the single learner instructional scenario). Secondly, they help to schedule the teaching and learning activities in design time, making to do further adjustments in run-time easily. Thirdly, they support students to get an alternative way of attending classes, as well as receiving personal guidance according to his/her assessments outcomes.

6) If we think in the long run, once we have been using the LD methodology for a substantial period of time, it should become possible to extract pedagogical patterns that help teachers identify the best teaching and learning practices, assisting us in the reporting and performance analysis. Although this is just a prediction, it clearly has great potential benefit that must be considered when adopting a corporative strategy for innovation purposes in educational practices.

\section{Conclusion}

The teachers involved in the design of the GDUS+ editor have concluded that the IMS-LD specification is a very useful methodological tool for formalizing the design of a pedagogical scenario in a higher education blended learning scenario.

We have discussed several usability related issues concerning eLearning tools arising from our experience designing a standards compliant tool. We have also described some of the lessons learned which might have wide applicability.

Further usability enhancements could come from a customizable user interface, because it may be useful to show or hide certain type of information according to the user profile; and for support for collaborative work.

\section{References}

[1] R. Koper., B. Olivier, "Representing the Learning Design of Units of Learning". Educational Technology \& Society, 7 (3), 2004, pp. 97-111.

[2] D. Griffiths, R. García-Robles et al, "Learning Design" Editor, eds. R. Koper and C. Tattersall, Springer-Verlag, 2005, pp. 109-135.

[3] D. Burgos, D. Griffiths, "The UNFOLD Project: Understanding and Using Learning Design", Open University of The Netherlands, 2005, pp. 25-30.

[4] R. Koper, "Current Research in Learning Design", IEEE Journal Educational Technology \& Society 9 (1), Special Issue on Learning Design, 2006, pp. 13-22.

[5] RELOAD: www.reload.ac.uk

[6] MOTPlus:

www.licef.teluq.uquebec.ca/gp/eng/productions/mot.htm

[7] D. Janetzko, "Dialogue-Based Authoring of Units of Learning", Proceedings of Sixth IEEE ICALT, 2006, pp. 436440 .

[8] X. Le Pallec et al., "Supporting generic methodologies to assist IMS-LD modelling", Proceedings of Sixth IEEE ICALT, 2006, pp. 923 - 927.

[9] E. Sánchez et al., "WebLD: A Web Portal to Design IMS LD Units of Learning", Proceedings of Seventh IEEE ICALT, 2007, pp. 880-881.

[10] Information model, pp 71, www.imsproject.org

[11] D. A. Norman, "The Psychology of everyday things", Basic Book, New York, 1998.

[12] L. L Constantine, L. A. D. Loockwood, "Software for use”, Addisson-Wesley, ACM Press, New York, 1999.

[13] J. Nielsen, "Usability Engineering", Academic Press, San Diego (USA), 1993.

[14] M. Good, T.M. Spine, J. Whiteside, and P. George, "User-derived impact analysis as a tool for usability engineering", Proceedings of $A C M C H I^{\prime} 86$, Boston (USA), 13-17 April 1986, pp. 241-246.

[15] J. Nielsen, R.L.Mack, "Usability Inspection Methods", John Wiley \& Sons, USA, 1994. 